\title{
Research on the SAR Image Target Tracking Algorithm Based on Sparse Representation and Dictionary Learning
}

\author{
Wenzhun Huang ${ }^{1, a^{*}}$ and Xinxin Xie ${ }^{1, b}$ \\ ${ }^{1}$ School of Information Engineering, Xining University, Xi'an 710123, China \\ ahuangwenzhun@xijing.edu.cn, b346148500@qq.com
}

Keywords: SAR image; Target tracking; Dictionary learning; Sparse representation; Algorithm

\begin{abstract}
In this paper, we conduct research on the SAR image target tracking algorithm based on the sparse representation and dictionary learning. The key technology of target tracking is to target can be extracted from the image detection, and to establish corresponding relationship with tracked target. Search target is extracted by testing on the whole image is very time consuming, in order to meet the requirements of real-time target tracking as should as far as possible to narrow the search scope in the entire image. By means of the criterion of correlation coefficient measurement to reflect the effect of target tracking, change according to the similarity of a certain strategy that is used to determine trace template, can effectively reduce the error updating the template. Under this background, our proposed methodology is feasible for enhancing the traditional approaches.
\end{abstract}

\section{Introduction}

In multi-sensor target tracking, the distributed fusion greatly reduced the calculation burden and the fusion center network communication between demands, have fast real-time processing ability, by the people's attention. Center fusion algorithm is the optimal fusion algorithm, therefore how to fusion algorithm by the distributed fusion algorithm reconstruction center is the result of the research in the recent years that caused many problems.

Target tracking based on template matching algorithm for the scene image quality is not high, can adapt to the target detection and tracking under complex scene that has the characteristics of strong anti-interference ability, thus in pattern recognition, target tracking, computer vision, and other fields has been widely applied. But the algorithm cannot adapt to intense illumination change and target deformation and keep out the influence of the interference factors. If template update can adapt to illumination changes and target deformation, while under the shade adopts the appropriate strategy to continue to track, then target tracking algorithm will greatly improve the adaptability and can meet the needs of practical engineering [1-3].

Sensor information flows from the target tracking system form and general integrated process level, multi-sensor tracking system is mainly centralized, distributed and hybrid as multistage structure. (1) In centralized multi-sensor tracking system, first of all, according to the basic target observation time successively to time integration of measurement point tracing, then for each sensor observations on the same target at the same time space fusion, it includes multi-sensor integrated the whole process of tracking and the state estimation. (2) In the distributed multi-sensor tracking system, the sensors first finish much of the single sensor target tracking and state estimation, which is completed time on the information fusion. (3) In multistage structure, the partial node can simultaneously or respectively is centralized, distributed and hybrid fusion center. They will be receiving and processing of data from multiple sensors or from multiple tracker track and system integration to again to various local fusion nodes transmit data to track correlation and fusion, which means the target inspection report passes through more than two levels of the location of the fusion processing [4].

Image reconstruction is one of the key issues in image and video processing. It from the image with noise, the purpose of the sample images, blurred image degradation such as the observation data in as much as possible to restore the real images. Group of the sparse aimed to sparse representation vector clustering and consider each group of sparse respectively used to reflect the local dependency between 
adjacent elements in sparse representation vector, through the process of updating the dictionary, to reflect the structure to the dictionary. In the training of dictionary model to join the group of sparse regularization, both to ensure that sparse representation signal sparse, and consider local dependency between adjacent said coefficient, and according to the properties of convex optimization theory of the monotone operators efficient algorithm that is given. Accordingly, we show the mentioned sample SAR image target tracking system as the following Fig. 1

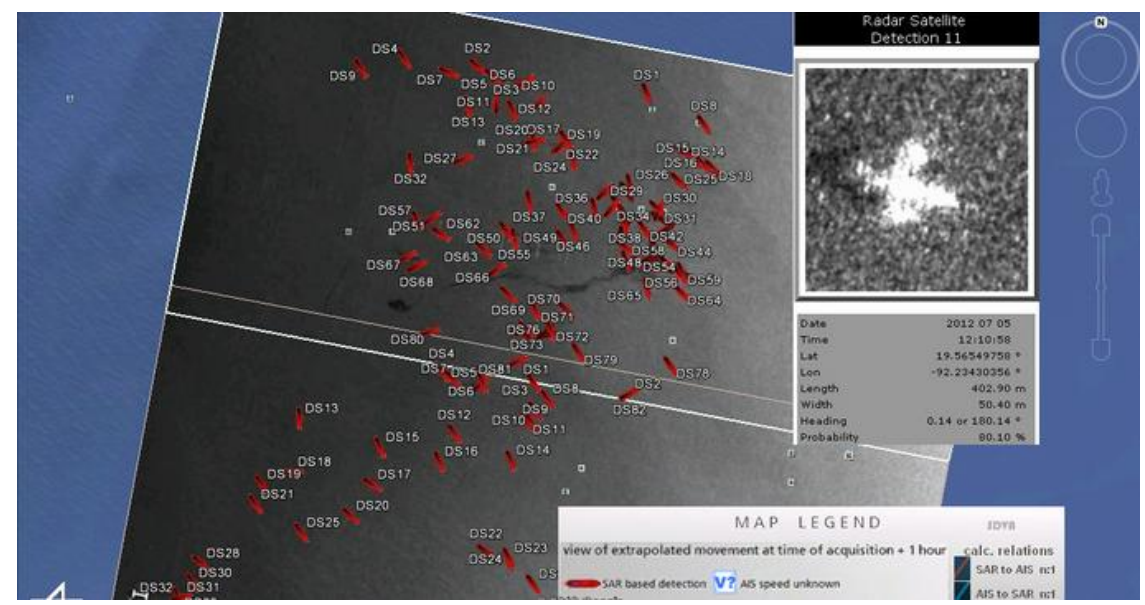

Figure 1. The Sample SAR Image Target Tracking System

In this article, we conduct research on the SAR image target tracking algorithm based on sparse representation and the dictionary learning. Target tracking problems are mainly concentrated on the image noise, illumination changes, complex background interference, and the characteristics of target is not stable, obscured, posture change, irregular movement, etc., to design a fast and robust target tracking algorithm is still challenging. In the later sections, we will show our methodology.

\section{The Proposed Methodology}

The Characteristics of the SAR Image. Radar image simulation is based on the ground, sensors, orbit parameters and radar imaging parameters, after the certain to scattering model, on the basis of in accordance with the principle of radar imaging, get the radar image simulation. Simulated images and real images will be in the image geometric features and radiation characteristics of consistency. In the case of space-borne SAR obtain booming, the space-borne SAR image simulation research has very important significance. In the 80s, the space-borne synthetic aperture radar and the real-time digital image processing technique into practical application and SAR has day, all-weather earth observation ability, has been widely used for the topographic mapping, exploration, environmental remote sensing and military and other fields. Although the SAR image has a wide application range, its application level is low, usage information is insufficient, the main reason is the accuracy level of the SAR image automatic information extraction is low that cannot satisfy actual demand of application department. How to get people from SAR image required for the reliable information is an important part of SAR technology research [5-6].

SAR image is gained by the literal way a single band of gray image, it reflects the features in the polarization mode of the band microwave backscatter features and is currently only depending on the image grey value of the SAR image to automatically extract the information of low accuracy, and the SAR image contains abundant texture information, different surface roughness shows characteristics of different texture, domestic and foreign scholars have used in information extraction of SAR image texture. Perception, on the surface of the texture of image region and describe the unique role, in order to make the texture analysis to get a better application in target recognition, people constantly looking for new algorithms to represent the texture of the area of the image, has formed a multitude of texture feature extraction method. In the layer sections, we will adopt the feature for further processing. 
The Image Sparse Representation. Sparse representation become a research hotspot recently, especially when the compression perception theory reveals that when the requested coefficient is thin enough, the minimum norm of NP problem can be converted to minimize norm problems to solve, sparse representation in machine vision, the machine learning, pattern recognition and other fields has caused wide attention. In view of computational complexity problem faced, this article puts forward a kind of simple and effective solution, its basic idea is still trying to seek a smaller than complete base to compute sparse representation of the test samples, so as to reduce the purpose of the computational complexity of the algorithm. The algorithm is the main idea is the result of data editing theory, which has nothing to do with the problem of data first cut, to a complex problem into a smaller range of local issues. In particular, the algorithm for each test sample first, determine its may belong to categories, and then use may be the samples belonging to class and not all of the training sample to the sparse representation the test sample and thus greatly reduced computational complexity algorithm.

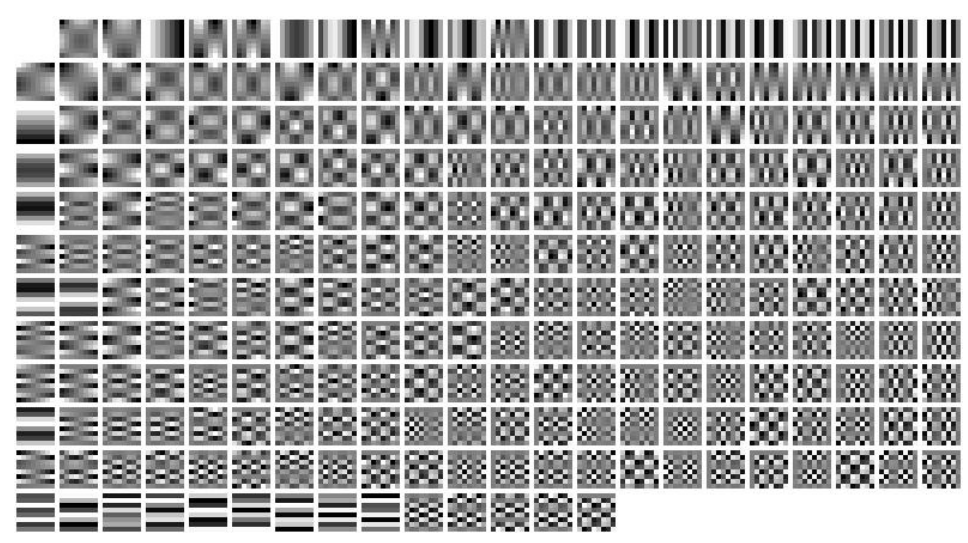

Figure 2. The Sparse Representation Sample Blocks

Using adjacent sample test sample and not all of training sample to compute sparse representation of the test sample, which to some extent reduced algorithm computational complexity, we could get the listed formula for expression.

$$
a_{i}=\arg \min \frac{1}{2}\left\|x_{i}-D a_{i}\right\|_{2}^{2}+\lambda\left\|a_{i}\right\|_{0}
$$

In sparse representation of image block is independent, independent between different image block sparse coefficient distribution the sparse degree of each other. Due to natural image information is of local redundancy, a high degree of similarity between different image block, so sparse representation and reconstruction, the use of the information of similarity between image block to restrain the local sparse coefficient. For the optimization work, we could express it as the follows.

$$
\min \|a\|_{0} \quad \text { s.t. } \quad\|D s-x\|_{2}^{2} \leq \chi
$$

When a shade, target the same position of the different characteristics will be affected, and the matching all block template should be activated. Therefore, dealing with same position of different characteristics of the corresponding block template corresponding coefficient imposes joint sparse regularization. The sparse coefficient constraint in the process of not only considers the similarity of each image block, also considering the similarity between the similar image block [7].

$$
x_{\text {reconstructed }}=D a=\left(\sum_{i=1}^{N} R_{i}^{T} R_{i}\right)^{-1}
$$

Based on this can the forenamed different frequency weighted conversion for multiple perspectives weighted problems and this article will introduce the technology to sparse representation of image fusion method, can get a new method of the adaptive weights learning. In order to better adaptive learning, improve the quality of the fused image, based on the mean and the average gradient two indicators to tentatively construct new multi-view weighted learning principles.

The Dictionary Learning Steps. For implicit in the image features can be obtained through sparse representation method study, the method in signal and image processing has obtained the very good 
effect, and is an effective image feature extraction algorithms, image sparse representation can learn a lot of local properties, such as the corner, edge features, and can obtain the higher resolution than the traditional adaptive method information, more in line with the human visual characteristics, which can express the image information more effectively.

Based on very complete dictionary of signal sparse decomposition is a new signal representation theory, it is made of super complete redundancy function system instead of the traditional orthogonal basis functions, for signal adaptive sparse extension provides great flexibility. Sparse decomposition can achieve data compression efficiency and more important is they can capture the signal using the redundancy feature of the dictionary inner nature as the following Fig. 3.
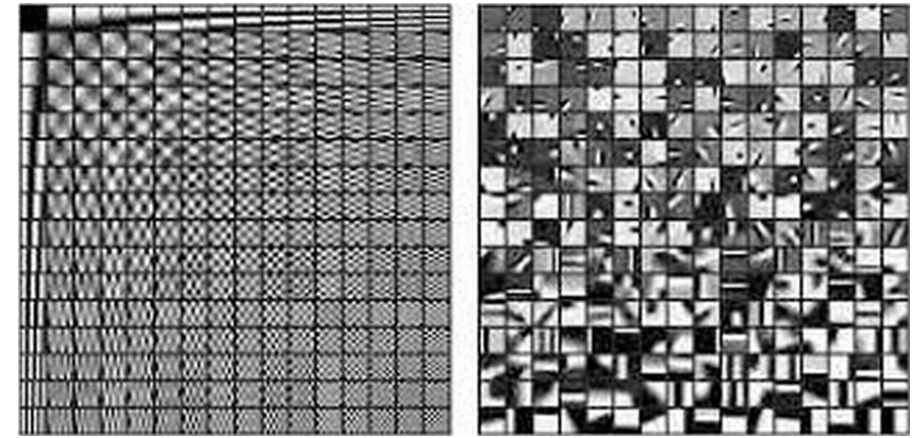

Figure 3. The Learned Dictionary for the Further Image Reconstruction

Signal sparse decomposition of the basic idea is: the use of super dictionary complete redundancy function as base functions, the choice of a dictionary as much as possible to meet by approximation signal structure, the elements in the dictionary are called atoms. Using the greedy algorithm and basic adaptive tracking algorithm, that found in the dictionary is the best linear combination of a few a few atoms to represent a signal and also known as the highly nonlinear approximation. For the better basic performance of the dictionary learning steps and procedures, we optimize it as follows.

$$
\begin{aligned}
& a=\min \|a\|_{0} \quad \text { s.t. }\|x-D a\|_{2} \leq \chi \\
& a=\min \|x-D a\|_{2} \quad \text { s.t. }\|a\|_{0} \leq \chi^{\prime}
\end{aligned}
$$

It is the core of the algorithm by introducing a thumbnail injective linear operator that using the projection imaging space of the linear operator do to promote thumbnail structure while optimize the dictionary. The algorithm combines the multiscale characteristic of wavelet transform and dictionary learning, in the domain of wavelet transform using the K training based on the block SVD dictionary, through the study of the core inverse wavelet transform of the dictionary to generate real multi-scale dictionary. Learn the dictionary at the same time show the multi-scale and the performance of the single scale. The inverse problems in image processing, this one element of the sparse feature is not enough to achieve good results. Clustering based on the sparse representation vectors and respectively considers each group of sparse to reflect local dependencies between elements sparse representation vector. In dictionary of learning process, the said dependencies between the vector elements reflect in the structure of the dictionary that can be expressed as the follows.

$$
J_{\text {general }}\left(a_{i}\right)=\sum \delta_{j}\left(B_{j} a_{i}\right)
$$

The Enhanced Target Tracking Algorithm. Particle filter is a kind of based on the recursive Bayesian estimation and filtering of Monte Carlo simulation method that can effectively deal with the nonlinear and non-Gaussian problems. Particle filter core idea is to use with a weight of particle sets approximate posterior probability density system, and then get the system state estimation.

The target deformation, there must be real image illumination changes, block, and so on and so forth, template is updated according to the actual image is the key to stable tracking. Choose a suitable template updating strategy, can to a certain extent, reduce the impact of these changes on the tracking effect. The normalized cross-correlation method of template matching tracking algorithm, through the establishment of normalized correlation function method for similarity calculation, and the template 
covers the following chart and target template error sum of squares of each pixel as their similarity measure which is expressed as the follows.

$$
D(i, j)=\sum_{m=1}^{M} \sum_{n=1}^{M}\left[S^{i, j}(m, n)-T(m, n)\right]^{2}
$$

How to share data, collaborate on data and information management node is a part of the track of the more important issues, such as which nodes involved in tracking, when to wake up in tracking, how to control the node transmit data and how long does it take to node communication, etc., all need comprehensive network environment to do that can be separated into the listed aspects.

- Under the condition of normal tracking, that compatibility should be higher than the update threshold. Template updating in each frame and save as backup template match the target template and replace the original backup template [8].

- When the matching degree is lower than the update threshold and higher matching threshold. Current template cannot very good tracking target, at this time because the target deformation, influence factors, such as basic illumination change, sunscreen, gray and contour of the target, causing the current template and the target matching degree is low, with better compatibility should be backup template as the target template for tracking.

- When object shade, the algorithm will match degrees below the matching threshold. At this time should stop updating and replacement of spare template, sparse representation method is used in filtering algorithm to estimate the location of the object, in a short period of time for target tracking, and will continue to detect the target and compare the backup template, until the target to appear, and the matching degree is higher than the threshold, to adopt the template matching algorithm and general perspectives.

\section{Conclusion}

In this paper, we conduct research on the SAR image target tracking algorithm based on the sparse representation and dictionary learning. Image a priori information model based on the general sparse representation has been widely used in the realization of the image tracking. In view of the sparse representation of dictionary selection and key issues of the coefficient estimates, based on the sparse representation is proposed combined with the local self-similarity of image reconstruction method. First through the block matching Euclidean distance finding similar image block, and use separate the similar image block set about dictionary local thinning and nonlocal sparse representation, in order to obtain more accurate coefficient of the sparse representation of sparse. Bergman iterative algorithm to solve the reconstruction model quickly and efficiently and uses the linear minimum mean square error estimation criterion to realize sparse coefficient estimates, in order to make sure to include the small coefficient of the detail of image texture of precise estimates. In the future, we will simulate proposed method to enhance the corresponding approaches that will be meaningful.

\section{References}

[1] Yang, Bo, and Ruoyu Yang. Interactive particle filter with occlusion handling for multi-target tracking. Fuzzy Systems and Knowledge Discovery (FSKD), 2015 12th International Conference on. IEEE, 2015.

[2] Savic, Vladimir, Henk Wymeersch, and E. Larsson. Target Tracking in Confined Environments with Uncertain Sensor Positions. (2015).

[3] Fang, Zicheng, et al. A fast implementation of Dynamic Programming based Track-Before-Detect for radar system. Radar Conference (RadarCon), 2015 IEEE. IEEE, 2015.

[4] Liu, Jiale, et al. Maneuvering Target Tracking with Radar/IR Heterogeneous Measurements. Journal of Aeronautics, Astronautics and Aviation 47.4 (2015): 369-376. 
[5] Zhang, Huanlong, et al. Visual tracking via constrained incremental non-negative matrix factorization. Signal Processing Letters, IEEE 22.9 (2015): 1350-1353.

[6] Papi, Francesco, et al. Generalized labeled multi-Bernoulli approximation of multi-object densities. Signal Processing, IEEE Transactions on 63.20 (2015): 5487-5497.

[7] Wisotzky, E., et al. Automated marker tracking using noisy x-ray images degraded by the treatment beam. Zeitschrift für Medizinische Physik 25.2 (2015): 123-134.

[8] Eslami, M., et al. Prediction of production of $22 \mathrm{Na}$ in a gas-cell target irradiated by protons using Monte Carlo tracking. Nuclear Instruments and Methods in Physics Research Section B: Beam Interactions with Materials and Atoms 342 (2015): 244-248. 